\title{
Les ambiguïtés sociales du progressisme pédagogique de John Dewey
}

\author{
Sébastien-Akira Alix
}

Résumé:

Nombreux sont les chercheurs qui inclinent aujourd'hui à s'intéresser principalement aux dimensions démocratiques du progressisme pédagogique de John Dewey ainsi qu'à son actualité pour questionner les débats sur l'école en France. La notion de progressisme pédagogique, particulièrement sous sa forme deweyenne, est ainsi souvent pensée et présentée comme étroitement solidaire, sinon consubstantielle, d'un processus de démocratisation en matière sociale. Le présent article vise à interroger cette interprétation en mettant en lumière les ambiguittés sociales du progressisme pédagogique deweyen au tournant des XIXe et XXe siècles aux États-Unis. En étudiant le rapport entre les choix politiques, philosophiques et éducatifs du philosophe, il montre qu'on ne peut pas considérer son progressisme pédagogique sans analyser, interroger et discuter les ambiguïtés sociales qu'il véhicule.

Mots-clés:

John Dewey; États-Unis; progressisme; théorie pédagogique; pratiques pédagogiques. 


\title{
The Social Ambiguities of John Dewey's pedagogical progressivism
}

\begin{abstract}
Many education researchers today tend to focus primarily on the democratic dimensions of John Dewey's pedagogical progressivism as well as its relevance to the ongoing debates on French schooling. The notion of pedagogical progressivism, especially in its deweyan form, is thus often viewed as closely intertwined, if not consubstantial, with democratic ideas and processes in social matters. This article aims at questioning this view by highlighting the social ambiguities of Dewey's pedagogical progressivism at the turn of the nineteenth and twentieth century in the United Sates. By studying the relationship between the philosopher's political, philosophical and educational choices, this article shows that one cannot consider Dewey's pedagogical progressivism without analyzing, questioning and discussing its inherent social ambiguities.
\end{abstract}

Key-words: John Dewey; United States of America; progressivism; pedagogical theory; pedagogical practice.

\section{As ambiguidades sociais do progressismo pedagógico do John Dewey}

Resumo: Muitos investigadores hoje tendem a estudar principalmente nas dimensões democráticas da progressividade educacional de John Dewey e em sua atual relevância para questionar os debates sobre a escola na França. A noção de progressismo pedagógico, particularmente na forma defendida por Dewey, é assim freqüentemente apresentada como intimamente interdependente, se não consubstancial, de um processo de democratização em questões sociais. O objetivo deste artigo é questionar essa interpretação, destacando as ambiguidades sociais do progressismo pedagógico do John Dewey na virada dos séculos XIX e XX nos Estados Unidos. Ao estudar a relação entre as escolhas políticas, filosóficas e educacionais do filósofo, este artigo mostra que não podemos considerar o progressismo pedagógico do Dewey sem analisar, questionar e discutir as ambiguidades sociais que ele transmite.

Palavras-chave: John Dewey; Estados Unidos da América; progressismo; teoria pedagógica; prática pedagógica.

\section{Las ambigüedades sociales del progresismo pedagógico de John Dewey}

Resumen: Actualmente, muchos investigadores se inclinan a estudiar principalmente las dimensiones democráticas del progresismo pedagógico de John Dewey y su relevancia para cuestionar los debates sobre la escuela en Francia. La noción de progresismo pedagógico, particularmente en su forma defendida por Dewey, a menudo se presenta como estrechamente interdependiente, si no consustancial, de un proceso de democratización en asuntos sociales. El propósito de este artículo es cuestionar esta interpretación al resaltar las ambigüedades sociales de la progresividad pedagógica deweyen en los comienzos de los siglos XIX y XX en los Estados Unidos. Al estudiar la relación entre las elecciones políticas, filosóficas y educativas del filósofo, este artículo muestra que no podemos considerar el progresismo pedagógico de John Dewey sin analizar, cuestionar y discutir las ambigüedades sociales que transmite.

Palabras claves: John Dewey; Estados Unidos de América; progresismo; teoría pedagógica; practica pedagógica. 
La pédagogie de Dewey n'a rien perdu de son actualité. Elle est actuelle, parce que la situation de nombreux pays francophones ressemble à beaucoup d'égards à celle dans laquelle Dewey conçut sa philosophie de l'éducation à Chicago à la fin du XIX siècle. Un pays en plein essor industriel, une population très diversifiée, de cultures et de langues différentes, rarement anglaises, une promotion sociale sans frontières de classe, rendaient caduques tout le système pédagogique hérité de la vieille Angleterre.(Deledalle, 1983, p. 44).

Ce mot de Gérard Deledalle, philosophe, traducteur et introducteur de l'œuvre pédagogique de John Dewey en France illustre de manière exemplaire la représentation du progressisme pédagogique américain, et spécialement deweyen, qui tend à dominer en France aujourd'hui. En effet, nombreux sont les chercheurs en sciences sociales et en sciences de l'éducation qui, à l'instar de Deledalle, inclinent à s'intéresser principalement aux dimensions démocratiques et émancipatrices du progressisme pédagogique de Dewey ainsi qu'à son actualité pour interroger les débats sur l'école en France au XXle siècle. À cet égard, on peut, par exemple, mentionner les travaux du sociologue Denis Meuret qui présente le système éducatif américain comme un modèle de l'empowerment, héritier direct de l'œuvre de Dewey, qui serait selon lui mieux adapté aux conditions nouvelles de l'enseignement en France que le système actuel, hérité de Durkheim (Meuret, 2007). De même, dans un ouvrage de 2014 intitulé La Pédagogie traditionnelle, le philosophe Jean Houssaye critique le fonctionnement de cette pédagogie qu'il dénonce; et regrette que l'héritage de l'éducation nouvelle, notamment celui de Dewey, n'ait pas suffisamment réussi à pénétrer le fonctionnement et les méthodes de l'école française d'aujourd'hui (Houssaye, 2014). Dans le même sens, quoique dans une perspective sensiblement différente, la philosophe et historienne des idées Joëlle Zask présente Dewey comme un pionnier et un précurseur de l'idée de "démocratie participative» pour lequel l'école «n'est pas l'ornement d'une société moderne démocratique, mais sa condition même de possibilité» (Zask, 2015, p. 4-5; Zask, 2001, p.64).

Cette interprétation qui insiste sur le caractère émancipateur et démocratique de la théorie pédagogique deweyenne s'enracine dans le fait que le philosophe a toujours présenté sa philosophie de l'éducation comme un moyen d'instaurer une démocratie progressiste conçue comme un processus infini de démocratisation. Dans son ouvrage majeur de 1916, Démocratie et éducation, Dewey écrit en effet que «le mélange à l'école de jeunes de races et de religions différentes ayant des coutumes distinctes crée pour tous un environnement nouveau et plus large»; qu' «un enseignement commun les accoutume tous à une unité de vue sur un horizon plus large que celui qui est visible pour les membres de n'importe quel groupe tant qu'il est isolé»; et qu'il est ainsi possible de «briser ces barrières de classe, de race et de territoire national qui empêchaient les 
hommes de percevoir la portée entière de leur activité. » (Dewey, 1916a, p.101 et 169). Aux États-Unis et en France, de semblables déclarations ont contribué à la formation autour de Dewey d'un large public d'enseignants, de chercheurs en sciences humaines et sociales et de pédagogues qui inclinent à considérer que le progressisme pédagogique du philosophe - particulièrement son expérience au sein de l'École Laboratoire de l'université de Chicago entre 1896 et 1904 - constitue une réponse adaptée aux problèmes que rencontrent les systèmes d'enseignement des sociétés occidentales démocratiques et multiculturelles du début du XXle siècle (Tanner, 1997; Meuret, 2011, p. 6, 20-25). La notion de progressisme pédagogique, particulièrement sous sa forme deweyenne, apparaît ainsi connotée positivement et est pensée comme étroitement solidaire, sinon consubstantielle, d'un processus de démocratisation en matière sociale. Ce point a bien été souligné par l'historien de l'éducation américaine David F. Labaree qui écrit :

\begin{abstract}
Aujourd'hui, progressisme signifie progressisme pédagogique. Cela veut dire baser l'instruction sur les besoins, les intérêts et les stades de développement de l'enfant; cela veut dire enseigner aux élèves les compétences dont ils ont besoin pour apprendre n'importe quel sujet, au lieu de se focaliser sur l'enseignement d'un sujet particulier; cela veut dire promouvoir la découverte et l'apprentissage par soi-même à travers la participation active de l'élève; cela veut dire avoir des élèves qui travaillent sur des projets correspondants à leur intérêt et qui intègrent les disciplines dans des thèmes socialement pertinents; et cela veut dire promouvoir les valeurs de communauté, de coopération, de tolérance, de justice et d'égalité démocratique. Dans les formules consacrées du jargon éducatif, on parle "d'enseignement centré sur l'enfant", "d'apprentissage par la découverte”, d'“apprendre à apprendre”. (Labaree, 2006, pp. 306-307)
\end{abstract}

Pour stimulante et légitime qu'elle puisse être, cette représentation séduisante du progressisme pédagogique américain, dont Dewey serait la figure de proue ou «Dieu le père» (Labaree, 2006, p. 310) - pour reprendre la formule de David F. Labaree -, a le défaut d'incliner au déracinement des idées du philosophe. En effet, elle tend à laisser de côté, d'un point de vue historique et théorique, la question de la pluralité de sens que peut recouvrir la notion de progressisme pédagogique dans l'œuvre de Dewey. Le présent article est consacré à l'analyse de cette pluralité de sens et, plus particulièrement, aux ambiguïtés sociales du progressisme pédagogique deweyen au tournant des $X I X^{e}$ et $X X^{e}$ siècles aux États-Unis. En prenant appui sur un certain nombre de travaux récents menés dans le champ de l'histoire de l'éducation aux États-Unis, le présent article met en lumière ces ambiguïtés de la philosophie de l'éducation de Dewey sous deux angles spécifiques: sous l'angle de la "race» ou de l'origine ethnique d'une part; et, d'autre part, sous l'angle social, en montrant que la pédagogie deweyenne est lestée d'ambiguïtés sociales qu'on ne saurait évacuer aisément. 


\section{Progressisme pédagogique et ségrégation raciale: un impensé deweyen}

En affirmant dès 1909 son rejet de l'idée qu'existerait une infériorité biologique entre les races, Dewey est à bien des égards l'un des réformateurs progressistes en éducation de son temps les moins conservateurs eu égard à cette question. Pour autant, dans son œuvre éducative aussi bien que politique, le philosophe n'appelle jamais à l'égalité des droits civiques des Africains-Américains et il reste étonnamment muet sur la question de la ségrégation raciale des écoles américaines; on ne trouve nulle part dans son œuvre une critique d'une telle séparation (Taylor, 2004). De tels silences ont conduit certains philosophes et historiens de l'éducation, comme Walter Feinberg et Frank Margonis, à soutenir que la conception deweyenne de l'éducation était constitutivement «racialisée», c'est-à-dire implicitement conçue pour les enfants blancs protestants de la classe moyenne américaine du tournant du XIXe et du XXe siècles aux États-Unis. Comme l'explique Frank Margonis,

La pédagogie révolutionnaire centrée sur l'enfant de Dewey fut développée avec des élèves européo-américains en tête, et par conséquent, les portraits de "I'élève" et de la "communauté de classe" (classroom community) qui se trouvent au centre de ses prescriptions pédagogiques sont - implicitement - européo-américaines et obscurcissent souvent les dynamiques de l'apprentissage pour les élèves de couleur au sein d'une société racialement polarisée (...) Les concepts centraux de la pensée éducative progressiste [deweyenne] font implicitement référence aux membres du groupe dominant. (Margonis, 2009, p. 19)

Dans cette perspective, Margonis affirme que les concepts clés de la pédagogie deweyenne devraient être abandonnés car ils seraient en réalité lestés de présupposés racistes qui conviennent mal à la démocratie américaine du XXIe siècle. Sans aller jusqu'à pareille extrémité, le présent article entend montrer, par l'analyse de plusieurs écrits du philosophe relatifs aux pratiques pédagogiques progressistes mises en œuvre dans différentes écoles des États-Unis, que le progressisme pédagogique de Dewey est porteur de certaines ambiguïtés eu égard au traitement pédagogique de certaines minorités ethniques qui traduisent un impensé deweyen s'agissant de la question raciale. À cet égard, dans leur ouvrage The Schools of To-Morrow de 1915, Dewey et sa fille Evelyn ne tarissent pas d'éloges sur les qualités de systèmes scolaires considérés comme progressistes à l'époque, allant jusqu'à ériger certains d'entre eux en modèles de réformes pour l'avenir. Or, précisément, ces «écoles de demain» s'inscrivent dans un contexte social caractérisé par une ségrégation raciale et scolaire de principe que Dewey semble considérer comme allant de soi. Par exemple, à propos de l'École Organique dirigée par Marietta Johnson à Fairhope dans l'Alabama, le philosophe et sa fille 
écrivent la chose suivante: "Madame Johnson tente une expérience dans des conditions qui sont celles des écoles publiques, et elle croit que ses méthodes peuvent être adoptées par tout système scolaire public. Elle ne demande presqu'aucune rétribution, et tout enfant est le bienvenu (any child is welcome)" (Dewey \& Dewey, 1915, p. 23). Or, précisément non; à l'époque, «tout enfant» n'est pas le bienvenu à l'École Organique car l'accès à cette institution est interdit à tous les enfants de couleur, notamment aux Africains-Américains. Comme l'a bien souligné I'historien Joseph W. Newman,

La ségrégation raciale était si largement acceptée dans la nation au début du vingtième siècle, que même John Dewey ne juge pas nécessaire de le signaler. Johnson avait des vues éclairées sur la race pour son temps, allant jusqu'à dénoncer la discrimination raciale dans ses discours et écrits. Certains des [membres] fondateurs de Fairhope non issus des États du Sud partageaient ses vues mais craignaient qu'elles compromettent leur expérience communautaire s'ils accordaient un traitement égal aux Noirs. Les Africains Américains vivaient incontestablement des vies séparées et non égales, et eu égard aux relations de race, l'École Organique maintenait le statu quo. (Newman, 1999, p. 74)

Ainsi, lorsque John et Evelyn Dewey écrivent que «tout enfant est le bienvenu» à Fairhope, ils excluent de l'expression «tout enfant» les jeunes Africains-Américains de l'époque. Une telle exclusion est révélatrice d'un impensé deweyen s'agissant de la question raciale. Cela apparaît d'autant plus clairement lorsqu'on considère l'analyse que le philosophe et sa fille proposent de l'école publique $n^{\circ} 26$ de la ville d'Indianapolis dans le huitième chapitre de leur ouvrage The Schools of To-Morrow. Dans cette école, "située dans un quartier pauvre, surpeuplé, habité par une population de couleur» et "ne compt[ant] que des élèves de couleurs", John et Evelyn Dewey sont frappés d'admiration pour le type d'éducation proposée aux jeunes garçons et filles noirs au sein de cette institution et qu'ils décrivent de la manière suivante :

Les garçons ont appris la menuiserie en fabriquant les objets dont on avait besoin dans l'école- tables, armoires et bibliothèques - et en faisant les réparations aux bâtiments. Les filles ont appris à coudre en confectionnant des vêtements pour elles, pour leurs frères et sœurs, ou en fabriquant des rideaux et des stores pour l'école. Elles ont appris à cuisiner en préparant des soupes en guise de repas chauds pour l'école et les voisins, et en cuisinant un repas complet pour leur classe. Outre les cours de couture et de cuisine, il existe pour les filles des classes de mode et de crochet. Ces deux classes sont organisées commercialement afin de permettre aux élèves de gagner quelque argent. (...) Le travail pour les garçons est organisé de même façon. Outre la menuiserie et les réparations, il y a une classe de cuisine pour les garçons, un département de cordonnerie et un atelier de couture (...) Dans l'atelier de réparation de chaussures, les garçons apprennent à réparer leurs chaussures. 
Le professeur est un cordonnier de profession, et les réparations doivent être bien faites.(Dewey \& Dewey, 1915, pp. 211-212)

Et le philosophe et sa fille d'ajouter qu'il s'agit dans cette école publique $n^{\circ} 26$ «d'apprendre aux [élèves] quels sont leurs vrais besoins», de leur «apprendre à gagner leur vie». Dewey applaudit ainsi dans ce passage une réforme pédagogique pour les jeunes Africains-Américains très proche de la conception restrictive de l'éducation professionnelle qu'il fustige à la même époque dans l'enseignement américain en général ; car, en effet, dans cette école publique $n^{\circ} 26$ d’Indianapolis, les élèves noirs étaient formés en vue d'occuper une position sociale subordonnée dans la société étatsunienne de l'époque en tant que domestiques, cordonniers, charpentiers, cuisiniers, etc. et, par là même, ils se trouvaient privés de possibilités réelles d'élévation sociale par l'école (Mohraz, 1979, p. 65-66; Feinberg, 1975, p. 117). Dewey va même jusqu'à souhaiter "que de semblables entreprises soient répétées dans toutes les grandes villes» du pays (Dewey \& Dewey, 1915, p. 207). Par là même, le philosophe approuve et légitime le type d'enseignement secondaire le plus souvent dispensé à l'époque aux jeunes Africains-Américains; type d'enseignement qui était très souvent conçu comme une formation professionnelle étriquée, à l'instar du programme scolaire mis en œuvre dans des institutions comme le Hampton Institute dans l'État de Virginie.

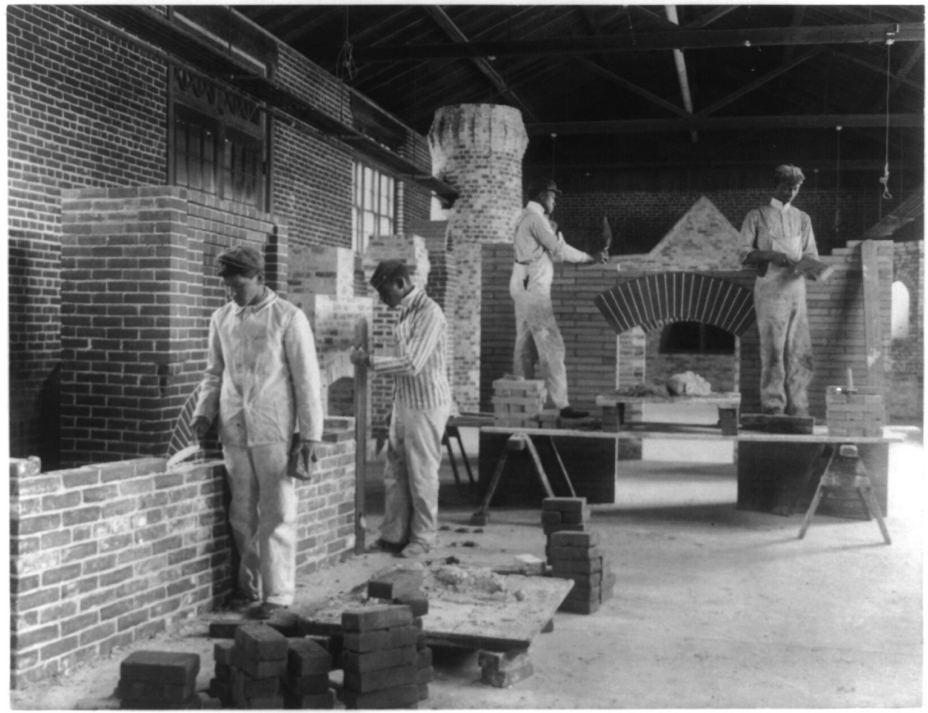

[Photographie 1. Une classe de maçonnerie, Hampton Institute, Virginia, ca. 1900.

Source: "Students in a bricklaying class, Hampton Institute, Hampton, Virginia»,

Frances Benjamin Johnston Collection, Library of Congress,

Prints and Photographs Division Washington, D.C. LC- USZ62-59187] 


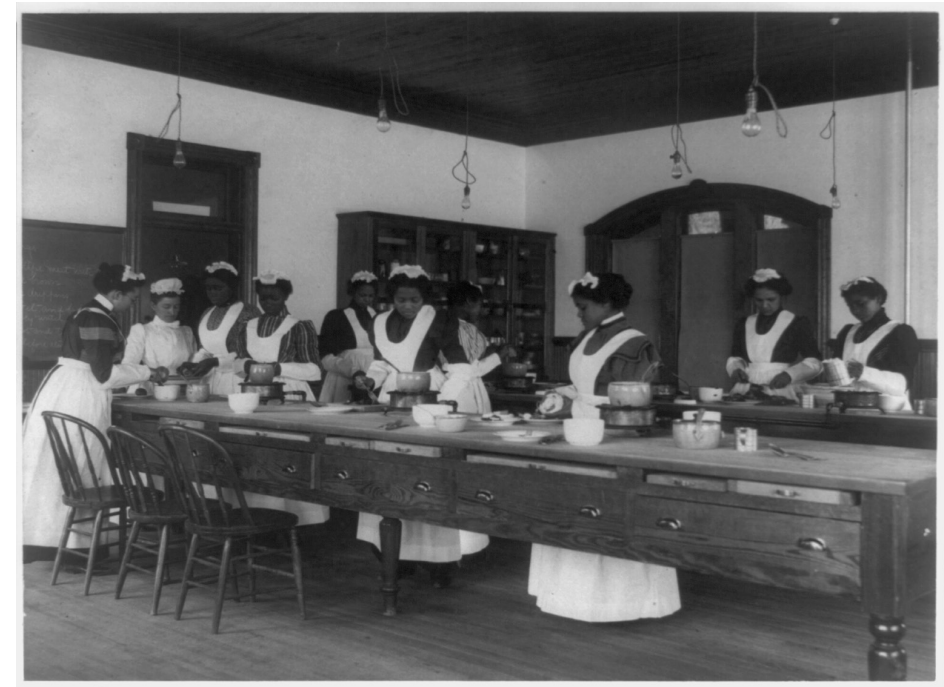

[Photographie 2. Jeunes femmes afro-américaines en classe de cuisine, Hampton Institute,

Virginia, ca. 1900. Source: «Ten Afro-American Women in Cooking at Hampton Institute, Hampton, Virginia», Frances Benjamin Johnston Collection, Library of Congress, Prints and Photographs Division Washington, D.C. LC- USZ62-59187]

Si Dewey semble de la sorte entériner le régime de ségrégation scolaire pour les Africains-Américains, on trouve dans son œuvre d'autres affirmations ambiguës à l'égard de groupes minoritaires à l'époque. À cet égard, dans un rapport sur les conditions des Polonais dans la Nation rédigé en 1918 par Dewey pour le département de la Guerre des États-Unis, le philosophe soutient l'idée que les travailleurs polonais doivent faire l'objet d'un processus d'américanisation par le biais d'une éducation adaptée dans le but de permettre à la nation américaine de conserver une main d'œuvre bon marché à l'issue de la Première Guerre mondiale. Dans ce rapport, le philosophe écrit la chose suivante:

La grande importance industrielle du travail polonais dans ce pays doit être gardée à l'esprit tout comme le fait qu'il y aura une pénurie de travailleurs après la guerre et qu'il y a déjà un mouvement à l'œuvre (qui devrait être soigneusement étudié) tendant à stimuler le retour des Polonais et d'autres individus nés dans les nations de l'Europe du Sud-Est dans leurs pays après la guerre. Avec la compétition commerciale vive qui aura nécessairement lieu après la guerre, toute tendance qui, d'un côté, contribue à dés-américaniser et, de l'autre, à renforcer l'allégeance [à leur pays d'origine] de ceux qui sont nés à l'étranger sur celle des États-Unis, mérite une grande attention.(Dewey, 1918, p. 73) 
À l'époque, ces différences de traitement proposées par Dewey - pour surprenantes et contradictoires qu'elles puissent paraître eu égard à ses déclarations démocratiques de principe - peuvent être expliquées par le fait que l'œuvre pédagogique deweyenne s'enracine dans un schème évolutionniste caractérisé par d'importantes dimensions ethnocentriques (Alix, 2017; Fallace, 2012, 2015). En effet, comme nombre de ses contemporains, Dewey souscrivait à l'époque à l'idée inspirée de l'évolutionnisme d'Herbert Spencer d'une analogie entre le développement de l'enfant et celui de la race humaine à travers l'histoire, faisant ainsi implicitement des membres des classes cultivées américaines le sommet du processus de civilisation (Gould, 1997). Dans ce cadre, les Africains-Américains et les immigrés polonais, parce que supposément situés à différents points le long du processus de civilisation font l'objet d'un traitement éducatif différent. Comme le souligne l'historien des idées Thomas D. Fallace,

En 1915, Dewey évaluait les Polonais-Américains et les Africains-Américains d'après
des normes différentes. Comme ses contemporains, Dewey croyait que ces deux
groupes pouvaient être placés sur une échelle unilinéaire du développement, les
Polonais se situant devant les Noirs. Les deux groupes étaient socialement déficients,
mais la distance culturelle entre l'immigrant polonais et l'Américain scientifique et
démocratique était suffisamment réduite pour être dépassée. Toutefois, la distance
pour les Africains-Américains était, à ce moment, trop grande.(Fallace, 2012b, p. 103)

À cet égard, s'il convient d'insister sur le fait que Dewey ne pose jamais en principe l'existence d'une infériorité constitutive ou biologique de l'esprit des membres des divers groupes ethniques qui composent le monde social de son époque, son œuvre pédagogique apparaît néanmoins porteuse d'une stratégie - largement inconsciente - d'instauration de différences hiérarchisantes entre les races et les cultures. D'où il découle que le progressisme pédagogique de Dewey apparaît porteur d'une ambiguïté foncière qui traduit un impensé deweyen s'agissant de la question raciale et ethnique. On retrouve d'ailleurs dans l'œuvre du philosophe une semblable ambiguïté s'agissant de l'articulation entre réforme scolaire et transformation de l'ordre économique et social.

\section{Le progressisme pédagogique de Dewey: une acceptation implicite et provisoire de l'ordre économique et industriel}

En France comme aux États-Unis, les chercheurs en éducation ont eu tendance à faire valoir la dimension réformiste et démocratique de l'œuvre pédagogique de Dewey en s'appuyant notamment sur son idée exprimée dès 1897 dans son Crédo pédagogique selon laquelle l'éducation est à concevoir comme «la méthode fondamentale du 
progrès social et de la réforme» (Dewey, 1897, p. 123). Commentant cette citation, la philosophe et historienne des idées Joëlle Zask écrit dans son Introduction à John Dewey la chose suivante:

Elle [L'école] ne destine pas l'élève à s'insérer dans la société en embrassant l'un des rôles nécessaires à son bon fonctionnement, mais à transformer les conditions sociales dans l'intérêt du renouvellement des interactions (...) L'éducation par l'expérience de Dewey et de sa Dewey's school n'est donc pas un détail. Elle est au contraire l'instrument le plus important de la reconstruction démocratique des publics. C'est à elle que revient la charge d'assurer ce point de contact entre l'expérience, l'esprit scientifique et les méthodes démocratiques que Dewey appelle: "extension socialisée de l'intelligence", "socialisation de l'intelligence", "organisation sociale de l'intelligence". (Zask, 2015, pp. 104-105)

En dehors des considérations relatives aux minorités évoquées plus haut, Dewey n'entend en effet jamais former l'élève en vue d'une fonction sociale existante ou «"adapter" les travailleurs au régime industriel existant» (Dewey, 1914, p.412), pour reprendre une formule du philosophe. Ce dernier cherche au contraire à offrir aux jeunes gens une éducation qui leur permette, d'une part, de percevoir les dimensions proprement culturelles et intellectuelles que recèlent les tâches qu'ils accompliront quotidiennement une fois qu'ils auront intégré le monde du travail; et, d'autre part, de mieux appréhender la nécessité et l'utilité de la division du travail en apprenant à coopérer et à travailler ensemble à la réalisation de projets communs. C'est au moyen d'une telle éducation que Dewey entend instaurer à terme une démocratie industrieuse dans laquelle les intérêts de l'individu et ceux de la société s'harmonisent parfaitement (Dewey, 1916a, p. 409).

Si, à l'instar de Joëlle Zask, de nombreux chercheurs ont ainsi souligné à juste titre le caractère proprement réformiste du progressisme pédagogique deweyen, ils n'ont, en revanche, pas mis en lumière les zones d'ombre que recèlent une telle position. Car, en effet, en concevant l'éducation comme «la méthode fondamentale du progrès social et de la réforme» et en se concentrant dans son œuvre sur la réforme de l'enseignement américain plutôt que sur celle de l'organisation de l'économie et du travail, Dewey tend au moins de manière implicite et provisoire à accepter l'ordre économique et industriel de la société états-unienne du tournant du XIXe et du XXe siècles. Ce point n'est nulle part mieux illustré que dans le passage suivant.

C'est parce que, hélas, l'ingénierie n'assure qu'une connexion formelle et non réelle entre la physique et le travailleur pratique dans les mines, que notre problème industriel est un problème éthique du genre le plus sérieux. La question du montant des 
rémunérations que le travailleur perçoit, du pouvoir d'achat de cette rémunération, des heures et conditions de travail sont, après tout, secondaires. Le problème s'enracine premièrement dans le fait que la science servant d'intermédiaire (mediating science) n'est pas connectée à sa conscience, mais simplement à ses actions extérieures. II n'apprécie pas la signification et la portée de ce qu'il fait; et il n'accomplit pas son travail du fait de sa participation à une conscience sociale et scientifique plus large. Si c'était le cas, il serait libre. Tous les accompagnements adéquats de salaire, d'heures, de conditions salubres et inspirantes lui seraient alors conférés (be added unto him), parce qu'il serait entré dans le royaume éthique.(Dewey, 1900, p. 325)

Dans ce passage, le philosophe met en lumière la dimension relativement conservatrice du point de vue social de sa philosophie et qui tient au fait qu'il situe principalement la liberté du travailleur du côté de la conscience et non des conditions matérielles de subsistance des individus. En effet, pour Dewey, les conditions de travail des employés, leur rémunération et le nombre d'heures qu'ils font ne sont pas les causes premières de l'aliénation ; cette cause est, au contraire, à situer premièrement du côté de leur non-participation à la "conscience sociale et scientifique plus large» qui est à l'origine des transformations du monde du travail, c'est-à-dire dans le fait que les «travailleurs pratiques» ne perçoivent pas les dimensions proprement intellectuelles que recèlent les tâches qu'ils accomplissent dans l'exercice de leur métier. Comme le philosophe l'explique,

L'industrie a cessé d'être une procédure essentiellement empirique, approximative (rule-of-thumb), transmise par la coutume. Sa technique est aujourd'hui technologique, c'est-à-dire qu'elle repose sur une machinerie résultant des découvertes en mathématiques, physique, chimie, bactériologie, etc. La révolution économique a stimulé la science en posant des problèmes à résoudre, en produisant un plus grand respect intellectuel pour les dispositifs mécaniques. Et l'industrie a été largement payée de retour par la science. En conséquence, les occupations industrielles ont un contenu intellectuel et des possibilités culturelles infiniment plus étendues qu'autrefois. La demande d'une éducation qui familiarise les ouvriers avec les bases et la portée scientifique et sociale de leur métier devient impérieuse, puisque ceux qui en sont privés sont inévitablement voués à jouer le rôle d'accessoires auprès des machines qu'ils font fonctionner.Sous l'ancien régime, tous les ouvriers qui exerçaient le même métier avaient à peu près la même connaissance et le même point de vue. La connaissance et l'ingéniosité personnelles pouvaient au moins s'exercer dans un domaine restreint, parce que le travail se faisait avec des outils dont l'ouvrier était directement le maître. De nos jours, l'ouvrier doit s'adapter à sa machine, au lieu d'adapter comme autrefois l'outil à ses propres fins. Si les possibilités intellectuelles 
de l'industrie se sont multipliées, les conditions industrielles tendent à rendre l'industrie moins éducative pour les masses qu'elle ne l'était du temps où l'on travaillait de ses mains pour des marchés locaux. C'est donc à l'école qu'incombe aujourd'hui la tâche de faire comprendre aux élèves les possibilités intellectuelles inhérentes à leur travail.(Dewey, 1916a, p. 407, tr. fr. ici modifiée)

Pour Dewey, c'est parce que les conditions de travail ne permettent plus aux ouvriers de saisir le contenu intellectuel et les possibilités culturelles de leur métier que l'école doit proposer un enseignement qui permette aux élèves de "reconnaître la valeur intellectuelle du travail» en articulant les éléments des sciences - physique, chimie, mathématiques, etc. - aux tâches industrielles concrètes (Dewey, 1916b, p. 413). En offrant de la sorte une éducation qui mette en perspective les activités industrielles ordinaires, le futur ouvrier aura à sa disposition les moyens intellectuels qui lui permettront de ne jamais «jouer le rôle d'accessoire auprès des machines qu'il fait fonctionner» et, partant, de se prémunir contre toute forme d'aliénation. Dans la perspective deweyenne, ce n'est qu'une fois que les travailleurs auront ainsi pleinement compris «la signification» et "la portée» de leur rôle - et qu'ils seront donc entrés dans le "royaume éthique» - que l'ensemble des éléments pratiques nécessaires à les libérer de toute aliénation «[eur] ser[ont] conférés».

En situant de la sorte le problème industriel principalement au niveau de la conscience, le philosophe pense ainsi pouvoir le résoudre en mettant en place une éducation qui permettra aux futurs travailleurs de saisir "les possibilités intellectuelles inhérentes à leur travail» (Dewey, 1916a, p. 407) sans pour autant attaquer les structures institutionnelles qui président à l'organisation de l'économie à la même époque. Par là même, Dewey se prive non seulement des moyens réels de transformer les maux de la société états-unienne qu'il décriait tant mais, s'il ne les légitime pas explicitement, les accepte - au moins provisoirement - en ce sens qu'il en diffère la réforme en en confiant la charge à la jeune génération dans un temps à venir. Ce point a d'ailleurs bien été mis en lumière par l'historien Harvey Kantor,

La foi de Dewey dans l'éducation pour réformer le monde du travail ne fut pas seulement le reflet de sa croyance dans le potentiel de la société américaine. Elle trahit également des préoccupations plus fondamentales. (...) Ce qui troublait Dewey (...) et d'autres progressistes libéraux n'était pas tant l'existence des classes que la distance croissante entre elles et les conflits que cela occasionnait. En conséquence, bien que leurs sentiments à propos du travail pointait vers une analyse de classe systématique de l'économie (a systematic class analysis of the economy), ces partisans de l'éducation professionnelle espéraient que le travail pourrait être réformé sans attaquer ceux qui "profitaient le plus du système existant". Ils proposèrent au lieu de cela de 
réformer le travail d'une manière rationnelle et ordonnée, et, comme de nombreux libéraux depuis lors, crurent que cela pourrait être fait en restructurant l'éducation dans le but d'éliminer graduellement, du mot même de Dewey, "les caractéristiques plus vastes et plus récalcitrantes de la société adulte”.(Kantor, 1988, p. 31)

En se concentrant ainsi sur la réforme de l'enseignement, Dewey maintient, au moins de manière implicite et provisoire, le statu quo industriel et économique. Cela apparaît d'autant plus clairement que le philosophe n'explique jamais comment les conditions matérielles "après tout secondaires" liées à l'exercice de leur travail seront accordées aux travailleurs dans les mines une fois que leur formation leur aura permis d'entrer dans "le royaume éthique», ni de quelle manière les richesses et les tâches devraient être réparties et organisées dans cette nouvelle société. En ne soufflant mot dans son œuvre sur la question de savoir comment les jeunes gens ainsi formés parviendront à transformer la structure sociale existante, le philosophe laisse impensée la question de l'articulation entre réforme scolaire et réforme de la société. Par là même, sa représentation de la bonne société démocratique et progressiste apparaît tout à la fois comme confuse et ambiguë; laissant le champ libre à une acceptation implicite de l'organisation de l'économie, du monde du travail et de l'ordre interne de la société démocratique industrielle et libérale du tournant du XIXe et du XXe siècles aux États-Unis.

Pour conclure, en France, la réception de l'œuvre de Dewey s'est historiquement inscrite dans une tradition d'histoire des idées tendant à s'intéresser principalement au côté émancipateur de l'éducation nouvelle; Dewey étant souvent présenté comme un des grands hérauts d'une éducation progressiste, démocratique et libératrice pour tous les enfants. Si une telle approche est louable en ce qu'elle a permis d'ouvrir un champ d'étude stimulant, elle court néanmoins le risque de déraciner les figures qu'elle entend étudier de leur contexte et, partant, de couvrir d'un voile certaines ambiguïtés sociales du progressisme pédagogique deweyen. Or, précisément, l'analyse de ces ambiguïtés peut permettre de nuancer et de compléter la représentation qui en est souvent donnée sans pour autant chercher à réduire ou minorer la profondeur indiscutable d'élaboration de l'œuvre éducative deweyenne. On ne peut toutefois pas considérer le progressisme pédagogique deweyen sans analyser, interroger et discuter les ambiguïtés sociales qu'il véhicule et qui sont liées au contexte de conceptualisation de l'œuvre du philosophe.

\section{Références}

Alix S.-A. (2017). L'éducation progressiste aux États-Unis. Histoire, philosophie et pratiques (18761919). Grenoble: Presses Universitaires de Grenoble.

Houssaye J. (2014). La Pédagogie traditionnelle. Une histoire de la pédagogie suivi de «Petite histoire des savoirs sur l'éducation». Paris: Éd. Fabert. 
Deledalle G. (1983). Présentation. In J. Dewey. Démocratie et éducation, suivi de Expérience et éducation. (pp.41-45).Paris: Armand Colin.

Dewey J. (1897).Mon Credo pédagogique. In G. Deledalle, John Dewey. Paris: PUF, 1995.

Dewey J. (1900). Psychology and Social Practice. Science, New Series, 11, 270,321-333.

Dewey J. (1914). Education vs. Trade-Training: Reply to David Snedden. In J. A. Boydston (éd.), (1976). The Middle Works of John Dewey, 8, Carbondale: Southern Illinois University Press.

Dewey J., Dewey E. (1915). The Schools of To-Morrow. New York: E. Dutton \& Company.

Dewey J. (1916a). Démocratie et éducation, suivi de Expérience et éducation. Paris: Armand Colin.

Dewey J. (1916b).The Need of an Industrial Education in an Industrial Democracy. Manual Training and Vocational Education, 17, 6, 409-414.

Dewey J. (1918).Conditions Among the Poles in the United States. Confidential Report. Washington, D. C.: Government Printing Office.

Fallace T. D. (2012). Dewey and the Dilemma of Race: An Intellectual History, 1895-1922. New York: Teachers College Press.

Fallace T. D. (2015). Race and the Origins of Progressive Education, 1880-1929. New York: Teachers College Press.

Feinberg W. (1975). Reason and Rhetoric: The Intellectual Foundations of 20th Century Liberal Educational Policy. New York: Wiley.

Gould S. J. (1997). La Mal-Mesure de l'Homme. Paris: Odile Jacob.

Kantor H. A. (1988). Learning to Earn. School, Work, and Vocational Reform in California, 1880-1930. Madison, Wisconsin: University of Wisconsin Press.

Labaree D. F. (2006). Progressisme, écoles, et education schools: une romance américaine. In R. Hofstetter, B. Schneuwly (dir.), Passion, Fusion, Tension. New Education and Educational Sciences - Éducation nouvelle et Sciences de l'éducation (End 19th-Middle 20th Century - Fin du 19e-milieu du 20e siècle). (pp.305-324). Berne: Peter Lang.

Margonis F. (2009). «John Dewey's Racialized Visions of the Student and Classroom Community». Educational Theory, 59, 1, 17-39.

Meuret D. (2007). Gouverner l'école. Une comparaison France/Etats-Unis. Paris: PUF.

Meuret D. (2011).Éducation, Démocratie, Espérance. In J. Dewey, Démocratie et éducation, suivi de Expérience et éducation. (pp.5-27). Paris: Armand Colin.

Mohraz J. J. (1979). The Separate Problem: Case Studies of Black Education in the North, 1900-1930. Westport, CT: Greenwood Press.

Newman J. E. (1999). «Experimental School, Experimental Community: The Marietta Johnson School of Organic Education in Fairhope, Alabama». In S. F. Semel et A. R. Sadovnik (éd.), "Schools of Tomorrow," Schools of Today: What Happened to Progressive Education. (pp.67-101).New York: Peter Lang. 
Tanner L.N. (1997). Dewey's Laboratory School: Lessons for Today. New York: Teachers College Press.

Taylor P. C. (2004).Silence and Sympathy: Dewey's Whiteness. In G. Yancy (éd.), What White Looks Like: African-American Philosophers on the Whiteness Question. (pp. 227-242). New York: Routledge,

Zask J. (2015). Introduction à John Dewey. Paris: La Découverte.

Zask J. (2001). L'élève et le citoyen, d'après John Dewey. Le Télémaque, 20, 53-64.

Sébastien-Akira Alix Chercheur associé au Centre de recherche sur les liens sociaux (CERLIS - UMR 8070) et enseignant au sein du département des Sciences de l'éducation de l'Université Paris Est Créteil Val-de-Marne. Ses recherches portent sur l'histoire et la philosophie de l'éducation aux États-Unis et en France ainsi que sur les circulations transnationales des savoirs en matière d'éducation. II est l'auteur de l'ouvrage L'éducation progressiste aux ÉtatsUnis. Histoire, philosophie et pratiques (1876-1919) paru aux Presses universitaires de Grenoble en 2017. Email: seb.alix@yahoo.fr

\section{Adresse \\ Sébastien-Akira Alix \\ Bâtiment Jacob \\ 45, rue des Saints-Pères \\ 75006 Paris}

Data de submissão: Março 2018

Data de avaliação: Agosto 2018

Data de publicação: Abril 2019 